\title{
CONTRACULTURAS: \\ UNA TIPOLOGIA \\ DE LA DELINCUENCIA
}

Doris Cooper

Puede definirse el concepto de Cultura, como "todo lo que se aprende socialmente y comparten los miembros de una sociedad" ". En términos generales, se compone de ideofacturas y manufacturas cuyas manifestaciones caracterizan en gran parte las sociedades en general y la nuestra en particular.

Por otro lado, la cultura a nivel de las ideofacturas comprende además de otros aspectos, un conjunto de normas y de valores característicos, y un conjunto de formas de comportamiento y de pautas de interacción que la identifican. Sin embargo, una Cultura también conlleva, en el caso de sociedades complejas, un conjunto de subculturas más o menos integradas y que constituyen subconjuntos relativamente homogéneos de aspectos socioculturales pautados, que aunque están relacionados con la cultura general de la sociedad, se distinguen de ella.

En este contexto conceptual, se ha intentado caracterizar sociedades a través de diferentes aspectos culturales y subculturales $y$, del mismo modo se ha intentado, con el análisis de la secuencia de manifestaciones culturales, la delimitación de secuencias evolutivas de la humanidad en general. En este último aspecto Spengle ${ }^{2}$ ha propuesto la existencia de ciclos evolutivos culturales que implican surgimientos y decadencias de conjuntos de sociedades, delimitados con la ayuda de análisis de fenómenos ideográficos y de manufacturas analizadas en su aspecto formal y simbólico.

En relación a nuestra Cultura particular, Escobar ${ }^{3}$ propone un análisis de

Horton y Hunt Sociologia. 1976.

Spengler La decadencia de Occidente. 1925.

Escobar R. Teoria de/ Chileno. 1981. 
manifestaciones particulares, intentando formular sus relaciones y fundamentos en características telúricas particulares. Este tipo de análisis es propuesto también por Spengler ${ }^{4}$ como un complemento de vital importancia para la correcta comprensión de las Culturas y su evolución, señalando que "falta igualmente la historia del paisaje -esto es de la flora, del suelo y del clima-en donde ha transcurrido la historia humana... pero la historia del hombre representa una tan dura pelea con la historia del paisaje, y permanece tan unida a éste por mil raíces, que sin la historia del paisaje no se comprenden bien la vida, el alma, el pensamiento"...

El análisis y la influencia del paisaje en nuestra Cultura chilena particular, son propuestos por Escobar, básicamente en su obra Teoría del Chileno. Destaca en ella que "si queremos entender cómo es la vida en Chile y por qué los chilenos son como son", ... debe considerarse el espacio geográfico y climático existente en nuestro suelo. Este espacio concreto $y$ distintivo, forja gran parte de nuestras características culturales.

Sin embargo, es nuestro interés destacar, además, que nuestra cultura, de génesis subsoleana, se caracteriza por presentar subculturas reconocidas y señaladas por diversos autores. Desde un punto de vista étnico, en primer lugar cabe subrayar la existencia de los mapuches, que caracterizan y representan un alto porcentaje de la novena región, existiendo además otras etnias de menor importancia numérica, pero que conforman también subculturas de relevancia. Por otra parte, se destaca la existencia de dos grandes subculturas: la Rural y la Urbana, cada una de las cuales presenta rasgos y complejos culturales peculiares.

Es reconocido el hecho de la existencia de fenómenos culturales particulares en cada una de estas dos macro-subculturas. Estos fenómenos culturales peculiares y distintivos en el ámbito rural, han sido definidos como "subsoleanos" a diferencia de la subcultura urbana que se caracteriza por concentrar una población con cultura "superficie", que suele recibir gran influencia de culturas extranjeras de países con mayor desarrollo tecnológico. Esta influencia implica el desarrollo de ciertos valores y metas-éxito diferenciales a las Rurales o subsoleanas.

Es básicamente Merton ${ }^{5}$ quien ha destacado e integrado en un marco teórico el concepto de meta-éxito. Postulamos la existencia de metas-éxito diferenciales en estas macro-subculturas Urbana y Rural en nuestro pais, las que hipotéticamente se derivan, en el primer caso, de la Cultura Occidental de tipo Industrial-Urbana y, en el segundo caso, de la Cultura subsoleana primigenia de nuestro país.

En la Teoría del Chileno, el autor distingue claramente dos subculturas diferenciales: es decir una "cultura bi-valente" ${ }^{6}$ aunque en el marco de un continuo-cultural, cuya entidad subyacente global es el carácter subsoleano.

Spengler. ob. cit. p. 60 Tomo 11.

Merton R. Teoría y Estructura Sociales. 1954.

Escobar R. ob. cit. p. 20. 
La cultura chilena, por ende comprende dos subculturas: la subsole (Rural) y la subsole-superficie (Urbana). El autor señala además específicamente la influencia relevante que sufre esta última subcultura de países extranjeros. Esta afirmación nos permite sugerir que esta influencia foránea proveniente básicamente de países de mayor desarrollo tecnológico, se traduce también en un conjunto de estructuras normativas y valóricas inmersas en la exportación e imitación de formas de vida y también de metas-éxito alternativas, en este caso de tipo urbano. Esta influencia es peculiar al sistema económico y cultural occidental, de modo que los países en vías de desarrollo se ven necesariamente inmersos en ella, pero principalmente en aquellos polos culturales-urbanos, que tienen mayor contacto como consecuencia de sus posiciones estratégicas desde un punto de vista comercial y socio-cultural.

Intentamos de esta forma, proponer la hipótesis de que en los centros urbanos de los paises occidentales, existen ciertos aspectos culturales comunes y entre ellos nos interesa destacar el aspecto valórico.

Si bien en las Urbes de los países Desarrollados predomina la meta-éxito económico como uno de los valores fundamentales que guían a los individuos en el logro de sus Status adquiridos, lo cual es derivado de los planteamientos de Merton relativos a la Cultura Norteamericana, este complejo cultural ha sido transmitido e impera también entonces en nuestros países en vías de desarrollo, como integrantes del sistema capitalista occidental. Deviene entonces, no sólo un intercambio comercial e industrial, sino también un intercambio cultural, en primer lugar, a nivel de las grandes urbes, el cual decanta tardiamente y probablemente en un futuro, en las zonas rurales más tradicionales.

Queremos destacar, cómo en nuestros países en vías de desarrollo, nuestra subcultura rural permanece aún en cierta medida no-contaminada de los fenómenos culturales urbanos de las macro-culturas de los países centrales de nuestro sistema occidental. Este fenómeno se revela por el análisis de diversos aspectos. Sin embargo intentaremos revisarlo analizando las características de la Delincuencia, como un fenómeno social de relevancia, que traduce aspectos culturales, subculturales y contraculturales.

Planteamos, entonces, que la Meta-éxito económico es una de las principales características a nivel valórico de las grandes Urbes de los países occidentales. En nuestra subcultura urbana la meta-éxito económico predomina también, de tal manera que en la medida que la estructura de Oportunidades presente características diferenciales a sectores marginales, se posibilita el surgimiento de formas de conducta de carácter Innovador Delictivo, que incluye a sectores de extrema pobreza y su necesidad de sobrevivencia.

Por otra parte, si como planteamos, nuestros sectores rurales conservan una subcultura relativamente tradicional y aun relativamente impermeable a estos tipos de valores predominantemente urbanos, concentrarán pautas relativas a metas-éxito-alternativas y diferenciales, que implican asimismo los fenómenos 
relacionados de consecución de prestigio aunque a través de metas alternativas subculturales de violencia machista, subculturas alcohólicas, folklores rural, e integración a grupos primarios contextuales.

Esta caracterización subcultural a nivel de metas-éxito sustentadoras del prestigio social, se manifiestan diversamente en variados fenómenos como consecuencia de su acentuación indiscriminada en situaciones extremas, de tal modo, que en el ámbito de la Delincuencia conforman elementos asociados y en cierta manera explicativos de tipos de Delincuencia Diferenciales.

Exponemos algunos antecedentes: La Delicuencia de las Urbes en nuestro país, asi como de las zonas Rurales, eran frecuentemente analizadas y expuestas en términos oficiales como homólogas, es decir, semejantes. Sin embargo, con un marco teórico, siguiendo básicamente a Merton, postulamos que, dadas las metaséxito diferenciales hipotéticamente existentes en áreas subculturales urbanas y rurales, era probable esperar Tipos de Delincuencia Diferenciales como resultado. Con una Investigación de carácter descriptivo, se constató que impíricamente eran distinguibles, un tipo de delincuencia urbano y un tipo de delincuencia rural, diferenciales además, según Sexo ${ }^{7}$.

El tipo de delincuencia urbana masculina se caracteriza por concentrar un $80 \%$ de "Delitos contra la Propiedad". Evidentemente es una manifestación contracultural de grupos de asociación diferencial ${ }^{8}, y$ de individuos aislados, que se encuentran inmersos en una subcultura particular caracterizada por metas-éxito económicas. El tipo de delito es por ende coincidente e hipotéticamente esperado. La delincuencia como fenómeno contracultural está enraizado en una subcultura específica y el tipo de manifestación debía ser correspondiente a un sistema de tipo urbano e industrial y corresponder además a las manifestaciones contraculturales explicitadas en las grandes urbes de los países desarrollados, en la medida de sus características universalizadoras de un tipo de cultura: internacional, capitalista, integradora y generalizante.

Por otra parte, nuestra subcultura rural, de características conservadoras tradicionales, presenta un tipo de contracultura diferencial que se manifiesta en un tipo de delincuencia masculina que concentra, comparativamente a la urbana, una alta proporción de "Delitos contra las Personas, la Familia y la Moral" $(50 \%)$, y como delito específico, una alta proporción de "Homicidios" (28\%). Las manifestaciones concretas de este tipo de delito, se vinculan con expresiones de formas de interacción social características de las zonas rurales y que conllevan manifestaciones o

7 Datos de Caracteristicas Sociodemográficas de la Criminalidad de Adultos en Chile. Cooper 82. Ver Datos en Anexo p. 10.

- La mayor parte de la Población Penal pertenece al Estrato Bajo.

8

Sutherland. Criminologia. 
consecuencias de las metas-éxito alternativas citadas. Estos hechos ocurren en situaciones de extrema violencia machista y consumo de alcohol e intervienen en su ejecución diversos elementos cortantes y otras herramientas de trabajo. Estas situaciones de extrema violencia implican una génesis subcultural particular, coincidente con el desarrollo teórico citado.

El tipo de delincuencia rural concentra, comparativamente a la urbana, una baja proporción de "delitos contra la Propiedad" (50\%) y cuyo delito específico predominante es el "Abigeato" y "Hurtos menores".

Esta subcultura rural y sus manifestaciones contraculturales particulares en los países en vías de desarrollo, se contraponen a las expresiones de las contraculturas rurales de los países desarrollados, donde los límites rural-urbanos han sido borrados por la implementación tecnológica y la consecuente inmersión de la subcultura urbana, conjuntamente a la internalización y práctica de metas alternativas de éxito económico. De tal modo que los tipos de delincuencia diferenciales urbanos y rurales de límites explícitos en nuestra Cultura Nacional, no se dan, o se dan en forma poco clara.

Como consecuencia, la modernización tiende a borrar los límites de estos tipos de delincuencia diferencial de lo cual dan cuenta las numerosas publicaciones del Departamento de Justicia Norteamericano ${ }^{9}$.

La modernización y el tradicionalismo de los ámbitos rurales y urbanos tienen además otras manifestaciones subculturales, esta vez relativas a los sexos, por la división básica del trabajo al interior de las sociedades, fundamentado en la asignación de tareas relativas al establecimiento de los Status Adscriptos, determina la formación y surgimiento de subculturas diferenciales también según sexo. Siendo la subcultura masculina urbana la generadora, en casos extremos, de manifestaciones contraculturales de tipo delictivo contra la propiedad, y la subcultura masculina rural la generadora de una contracultura, en casos extremos una conducta delictiva que se caracteriza por los "Homicidios", encontramos que, en el caso del sexo femenino, las manifestaciones contraculturales son también diferenciales.

El sexo femenino se caracteriza por sustentar una subcultura basada en su Status Adscripto, el cual se fundamenta en la asignación de tareas de crianza, socialización y labores domésticas generales. Su Status, a diferencia del sexo masculino, es adscripto también, en primer lugar, al hogar de origen, y en segundo lugar al status del cónyuge, en cuyo caso se anexa además el rol de sumisión y complacencia sexual conyugal.

Como consecuencia, los conflictos que desencadenan manifestaciones delictuales se relacionan con otra meta-éxito alternativa, en este caso de tipo femenino y que se relaciona con los valores de madre y esposa.

9 Entre otros: J. Warner Jr. Rural Crime, Rural Crimina/s, Rura/ Delinquents: Past Research and Future Directions en Juvenile Justice in Rural América. U.S. Department of Justice. 1980. 
Los aspectos contraculturales del sexo femenino en el ámbito rural, se manifiestan en altas proporciones de "Delitos contra las Personas la Familia y la Moral" $(74 \%)$ típicamente femeninos como el "Parricidio" (50\%) (al cónyuge, hijos o padres), "Homicidios" (a víctimas vinculadas afectivamente), "lesiones" (a los hijos), "corrupción de menores", "abortos", "infanticidios", etc. Entre estos delitos predomina como delito específico el "Conyugicidio". En este ámbito, como consecuencia de aspectos contraculturales del machismo y violencia del sexo masculino, en contraposición a sus metas y valores femeninos elementales. En casos de extrema violencia, la mujer opta, dados los bajísimos recursos y acceso a otros medios legales, a la eliminación del cónyuge, considerando los continuos malos tratos físicos y morales, en un ambiente habitualmente alcoholizado. Esta manifestación contracultural se enraíza en la subcultura tradicional rural de nuestro medio. Su manifestación es un resultado de la concatenación subcultural rural.

En el ámbito urbano, la delincuencia femenina también se caracteriza por una alta proporción de "Delitos contra las Personas, la Familia y la Moral", específicamente femeninos $(51 \%)$. Sin embargo, los conflictos desencadenadores son diferenciales: el delito predominante se relaciona con el "parricidio" como delito específico, de los hijos. Este fenómeno se enraíza en un contexto en el que los valores sociales sancionan fuertemente a las madres solteras, induciendo, en casos de extrema desesperación, a la eliminación de la causa del conflicto. El aislamiento, a causa de que en un alto porcentaje se trata de migrantes rural-urbanas que trabajan como domésticas, y el rechazo social probable generan el conflicto particular, que sólo puede darse en esta subcultura urbana a la cual se tratan de integrar. La meta-éxito-económico como valor urbano implica necesariamente el alcanzar un trabajo estable y éste sólo podría ser logrado sin familia.

Es además destacable la internalización progresiva del meta-éxito-económico, urbano y básicamente masculino. Surge así una nueva proporción de "Delitos contra la Propiedad" que alcanza un $49 \%$, caracterizada por una alta asociación a grupos delictuales dirigidos por varones.

Hemos distinguido, en esta forma, cuatro tipos diferenciales de delincuencia y hemos intentado relacionarlos con sus subculturas de origen.

Sin embargo, nos queda además que plantear otro tipo de delincuencia diferencial a los anteriores, y más que producto de una subcultura particular, del fenómeno de aculturación.

En relación al fenómeno de Aculturación o choque subcultural se generan, en casos extremos, dos tipos más de delincuencia. En primer lugar, el de los migrantes rural-urbanos y el de los mapuches.

En el caso de los migrantes rural-urbanos, se esperaba encontrar hipotéticamente, en base al marco teórico señalado, un tipo de delincuencia intermedio al tipo rural y al tipo urbano - ya expuestos-, relativos al sexo masculino. Es decir 
proporciones intermedias de "delicuencia coitra la Propiedad" y "contra las Personas, la Familia y la Moral" y delitos específicos correspondientes. Sin embargo, su expresión empírica fue, para nuestra sorpresa, similar a la manifestada por la contracultura de tipo rural. Es decir predominio de "Delitos contra las Personas" y específicamente, "Homicidios". La explicación e inferencia teórica implican el considerar que los migrantes se trasladan a las urbes sólo una vez que han internalizado la subcultura rural, e implica además que el fenómeno de aculturación no es de gran magnitud, por migrar en edades relativamente adultas. Como consecuencia, sus manifestaciones delictivas, conllevan los parámetros rurales.

En el caso de los mapuches, en cambio, el fenómeno de la aculturación es bastante mayor. La Cultura Mapuche es una totalidad cultural diferente y totalizadora, además de rural. El choque cultural es de gran magnitud y los efectos del prejuicio y segregación a nivel urbano, en el caso de los migrantes rural-urbanos de origen mapuche, conllevan un conflicto de gran violencia. Estos aspectos generan, en casos extremos, delitos de alta gravedad penal: "Homicidios" reiterados con agravantes, resultantes de un gran cúmulo de frustraciones y situaciones de extrema violencia y desamparo. El delito específico de "Homicidio" alcanza un $80 \%$ del total de los delitos ${ }^{10}$.

Hemos intentado destacar de qué manera, los actos delictivos individuales, conforman expresiones sociales características contraculturales, ligadas y resultantes de situaciones extremas conflictivas que, sin embargo, se encuentran íntimamente ligadas a la subcultura de origen. Las expresiones individuales lo grupales) delictivas, corresponden a patrones sociales resultantes de subculturas rurales y urbanas $y$ de subculturas, inmersas en estas subculturas complejas diferenciales según sexo. Estas características subculturales conforman además nuestra Cultura Nacional, diferencial en sus límites claramente demarcados, al caso de los países más desarrollados. Por otra parte hemos destacado el fenómeno de la contracultura mapuche, como consecuencia del conflicto de aculturación, distinto de aquel que enfrentan los migrantes rural-urbanos.

Nuestras características delictuales son particulares también, y relativas a nuestro nivel de desarrollo y modernización. Es evidente como aún en la expresión delictiva, mantenemos características aún diferenciales entre zonas urbanas y rurales y según sexo, aspecto que en los países desarrollados ya no se diferencia en forma tan clara.

Hemos intentado de este modo destacar la coherencia entre la tipología delictual empírica presentada y los aportes teóricos de los autores mencionados, los cuales nos permitieron en una primera etapa, hipotetizar acerca de las características de nuestras contraculturas y en una segunda aproximación, dar cuenta de la

- Datos de una Investigación Exploratoria realizada en el C.R.S. Temuco. Cooper. 
profunda imbricación entre sus teorías y nuestra realidad empírica en algunos de los aspectos de mayor relevancia.

Es así como el marco teórico de Merton permite predecir, principalmente, el tipo de contracultura asociada a las grandes urbes, la cual expuesta básicamente en función de los Estados Unidos pasa a ser posible de aplicar a nuestras urbes, a través de la consideración teórica de la subcultura subsole-superficie desarrollada por Escobar.

Por otra parte, los planteamientos de Escobar y Zamorano-Munizaga dan cuenta de los principales aspectos subculturales rurales y subsoleanos, a cuyas características es posible asociar y predecir hipotéticamente otro tipo de manifestaciones contraculturales específicas, diferenciales.

Finalmente, los aspectos relativos a las manifestaciones contraculturales de migrantes rural-urbanos, del sexo femenino y mapuches, aunque no son directamente inferibles de los planteamientos teóricos señalados, prestan en conjunto gran utilidad en la búsqueda de planteamientos hipotéticos que permiten, finalmente, lograr una descripción empírica fundamentada en los macro-aspectos teóricos señalados.

Ha sido nuestro interés relacionar aspectos contraculturales, subculturales y culturales nacionales y destacar así manifestaciones de coherencia entre estos distintos niveles conceptuales. Por otra parte, destacar la relevancia de los fenómenos de modernización y tradicionalismo y sus implicaciones, a nivel de las concreciones empíricas de las contraculturas nacionales. Aunque los aspectos delictivos no forman parte, en términos estrictos, de una manifestación "humana positiva", son parte de la Cultura como fenómeno contracultural. 
TIPOS DE DELINCUENCIA:

ALGUNAS CARACTERISTICAS DELICTUALES

Delitos Tipo I y II: Contra las Personas, la Familia y Moral;

Tipo III: Contra la Propiedad

\begin{tabular}{|c|c|c|c|c|c|}
\hline & $\begin{array}{l}\text { Tipos de } \\
\text { Delitos }\end{array}$ & $\begin{array}{l}\text { Delitos } \\
\text { Predominantes }\end{array}$ & Reincidencia & Habitualidad & $\begin{array}{c}\text { Ultimo Delito } \\
\text { Grupo } \\
\text { Delictual }\end{array}$ \\
\hline Población & $\begin{array}{l}\text { Delitos }|-1| \\
=48 \%\end{array}$ & $\begin{array}{l}\text { Homicidio } \\
=28 \%\end{array}$ & $\begin{array}{l}\text { Primerizos } \\
=51 \%\end{array}$ & $\begin{array}{l}1 \text { Delito }=31 \% \\
2 \text { y más }=69 \%\end{array}$ & $\begin{array}{c}\text { Delito I y II } \\
\text { solo }=64 \% \\
\text { en gra. }=36 \%\end{array}$ \\
\hline & Delitos III & Abigeato & Reincidentes & (Hasta 2 Delitos & Delito III \\
\hline Masculina & $=52 \%$ & $=14 \%$ & $=49 \%$ & $=70 \%$ & $\begin{array}{l}\text { solo }=\mathbf{4 4} \% \\
\text { en gr. }=\mathbf{5 5} \%\end{array}$ \\
\hline Migrantes & $\begin{array}{l}\text { Delitos I-II } \\
=44 \%\end{array}$ & & $\begin{array}{l}\text { Primerizos } \\
=44 \%\end{array}$ & 1 Delito $=15 \%$ & $\begin{array}{l}\text { Delito I y || } \\
\text { solo }=71 \%\end{array}$ \\
\hline R-U (4 Prov.) & & $\begin{array}{l}\text { Homicidio } \\
=22 \%\end{array}$ & & & en gr. $=29 \%$ \\
\hline Masculinos & $\begin{array}{l}\text { Delitos III } \\
=56 \%\end{array}$ & & $\begin{array}{l}\text { Reincidentes } \\
=56 \%\end{array}$ & 2 y más $=85 \%$ & $\begin{array}{l}\text { Delito III } \\
\text { solo }=66 \% \\
\text { en gr. }=36 \%\end{array}$ \\
\hline Migrantes & $\begin{array}{l}\text { Delitos I-II } \\
=45 \%\end{array}$ & & $\begin{array}{l}\text { Primerizos } \\
=43 \%\end{array}$ & 1 Delito $=16 \%$ & $\begin{array}{l}\text { Delito I y } \| \\
\text { solo }=83 \%\end{array}$ \\
\hline R-U (Otras Prov.) & & $\begin{array}{l}\text { Homicidio } \\
=23 \%\end{array}$ & & & en $\mathrm{gr} .=17 \%$ \\
\hline \multirow[t]{2}{*}{ Masculinos } & $\begin{array}{l}\text { Delitos III } \\
=55 \%\end{array}$ & & $\begin{array}{l}\text { Reincidentes } \\
=57 \%\end{array}$ & 2 y más $=84 \%$ & $\begin{array}{l}\text { Delito III } \\
\text { solo }=45 \% \\
\text { en gr. }=55 \%\end{array}$ \\
\hline & $\begin{array}{l}\text { Tipos de } \\
\text { Delitos }\end{array}$ & $\begin{array}{c}\text { Delitos } \\
\text { Predominantes }\end{array}$ & Reincidencia & Habitualidad & $\begin{array}{c}\text { Ultimo Delito } \\
\text { Grupo } \\
\text { Delictual }\end{array}$ \\
\hline $\begin{array}{l}\text { Pob. Penal } \\
\text { Urbana }\end{array}$ & $\begin{array}{l}\text { Delitos I-II } \\
=21 \%\end{array}$ & $\begin{array}{l}\text { Robo } c / v \\
=20,3 \%\end{array}$ & $\begin{array}{l}\text { Primerizos } \\
=\mathbf{5 4} \%\end{array}$ & $\begin{array}{l}1 \text { Delito }=22 \% \\
2 \text { y más }=78 \%\end{array}$ & $\begin{array}{l}\text { Delito I y } \| \\
\text { solo }=67 \% \\
\text { en gr. }=33 \%\end{array}$ \\
\hline $\begin{array}{l}\text { Gran Santiagol } \\
\text { Masculinos }\end{array}$ & $\begin{array}{l}\text { Delito III } \\
=79 \%\end{array}$ & $\begin{array}{l}\text { Robo c/f. } \\
=19,6 \%\end{array}$ & $\begin{array}{l}\text { Reincidentes } \\
=46 \%\end{array}$ & $\begin{array}{l}\text { (Hasta } 2 \text { Delitos } \\
=48 \%)\end{array}$ & $\begin{array}{l}\text { Delito III } \\
\text { solo }=46 \% \\
\text { en gr. }=54 \%\end{array}$ \\
\hline $\begin{array}{l}\text { Pob. Penal } \\
\text { Femenina }\end{array}$ & $\begin{array}{l}\text { Delitos } 1-11 \\
=74 \%\end{array}$ & $\begin{array}{l}\text { Parricidio } \\
=50 \%\end{array}$ & $\begin{array}{l}\text { Primerizas } \\
=78 \%\end{array}$ & 1 Delito $=70 \%$ & $\begin{array}{l}\text { Delito I y } \| \\
\text { sola }=68 \% \\
\text { en gr. }=32 \% \\
\end{array}$ \\
\hline Rural & $\begin{array}{l}\text { Delitos III } \\
=26 \%\end{array}$ & & $\begin{array}{l}\text { Reincidentes } \\
=22 \%\end{array}$ & 2 y más $=30 \%$ & $\begin{array}{c}\text { Delito III } \\
\text { sola }=58 \% \\
\text { en gr. }=42 \%\end{array}$ \\
\hline $\begin{array}{l}\text { Pob. Penal } \\
\text { Femenina }\end{array}$ & $\begin{array}{l}\text { Delitos }|-1| \\
=51 \%\end{array}$ & $\begin{array}{l}\text { Parricidio } \\
=26 \%\end{array}$ & $\begin{array}{l}\text { Primerizas } \\
=64 \%\end{array}$ & 1 Delito $=56 \%$ & $\begin{array}{l}\text { Delito } \mid \text { y } \| \\
\text { sola }=76 \% \\
\text { en gr. }=24 \% \\
\end{array}$ \\
\hline Urbana & $\begin{array}{l}\text { Delitos III } \\
=49 \%\end{array}$ & & $\begin{array}{l}\text { Reincidentes } \\
=36 \%\end{array}$ & 2 y más $=44 \%$ & $\begin{array}{c}\text { Delito III } \\
\text { sola }=39 \% \\
\text { en gr. }=61 \%\end{array}$ \\
\hline
\end{tabular}




\section{BIBLIOGRAFIA}

bastide, R., Antropología Aplicada. Amorrortu. 1972.

COOPER, D., Características Sociodemográficas de la Criminalidad de Adultos en Chile. 1982.

DEPARTMENT OF JUSTiCE. U.S., Juvenil Justice in Rural America. 1980.

DePARTMENT OF JUSTICE. U.s., The Urban Public Sector and Urban Crime: A Simultaneous System Approach. 1980.

escobar, R., Teoría del Chileno. Corporación de Estudios Contemporáneos. 1981.
HORTON Y HUNT, SOciología. 1979.

MERTON, R., Teoría y Estructura Sociales. F.C.E. 1964.

SPEngleR, o., La Decadencia de Occidente. 1925.

Sutherland y Cressey, Principios de Criminología. 1966.

zamorano y munizaga, Crimen y Alcohol. 1963.

U. de Chile. 